\title{
ADMISSÃO DE PACIENTES COM ACIDENTE VASCULAR CEREBRAL EM HOSPITAL PÚBLICO
}

\section{ADMISSION OF PATIENTS HAVING A STROKE IN PUBLIC HOSPITAL}

Luciana Protásio de Melo (Orcid: 0000-0003-3320-9428)

Débora Carvalho de Oliveira (Orcid: 0000-0003-1130-802X) ${ }^{1}$

Ana Amália Torres Souza Gandour Dantas (Orcid: 0000-0003-1264-6450)

Renan Alves da Silva Júnior (Orcid: 0000-0002-5393-3088)

Tatiana Souza Ribeiro (Orcid: 0000-0002-9611-1076) ${ }^{2}$

Tania Fernandes Campos (Orcid: 0000-0002-9906-3292)

Contato

Luciana Protásio de Melo

E-mail: protasio.melo@yahoo.com.br

${ }^{1}$ Departamento de Fisioterapia, Universidade Federal do Rio Grande do Norte, Rio Grande do Norte, Brasil ${ }^{2}$ Faculdades Integradas de Patos, Paraíba, Brasil.

Órgão financiador: Conselho Nacional de Desenvolvimento Científico e Tecnológico $(\mathrm{CNPq})$ por meio do processo no $409797 / 2006-5$.

\section{RESUMO}

Introdução: o Acidente Vascular Cerebral (AVC) é um importante problema de saúde pública. Uma parcela signifativa dos óbitos e das sequelas graves poderia ser evitada com medidas preventivas ou com prestação de socorro de forma correta e rápida. Objetivo: avaliar os pacientes com AVC na chegada ao hospital público e verificar as condutas adotadas no primeiro atendimento. Método: participaram do estudo 433 pacientes, que foram avaliados por meio do instrumento Step 1. Os dados foram analisados pelo teste Qui-quadrado. Resultados: verificou-se maior frequência de casos na faixa etária de 70 anos a 89 anos $(40,9 \%)(p=0,001)$ e de indivíduos sem escolaridade $(41,2 \%)(\mathrm{p}=0,001)$. Os pacientes chegaram ao hospital dentro de 24 horas do início da doença $(63,3 \%)(\mathrm{p}=0,001)$, e o tempo de realização do primeiro exame também se deu dentro de 24 horas $(85,9 \%)(\mathrm{p}=0,001)$. Conclusão: a hipertensão arterial sistêmica, o AVC isquêmico, a dependência funcional moderada, a forma de condução ao hospital e o tempo de chegada sugerem que os pacientes com AVC podem não estar dentro dos requisitos da janela temporal para que se institua uma terapêutica adequada para o controle e a evolução do ictus, o que pode indicar a necessidade de políticas públicas que contribuam para o aperfeiçoamento da rede SUS, na sua linha de cuidado ao AVC.

Palavras-chave: Acidente Vascular Cerebral; Epidemiologia; Vigilância em Saúde Pública; Educação em Saúde.

\begin{abstract}
Introduction: strokes are an important public health problem. A significant proportion of deaths and serious sequelae could be prevented with preventive or corrective measures. Objective: to evaluate the patients who had a stroke on their arrival at the public hospital and to verify the behaviors adopted in the first care. Method: A total of 433 patients participated in the study, which were evaluated using the Step 1 instrument. Data were analyzed by Chi-square test. Results: there was a higher frequency of cases in the age group of $70-89$ years $(40.9 \%)(p=0.001)$ and of individuals without schooling $(41.2 \%)(\mathrm{p}=0.001)$. Patients arrived at the hospital within 24 hours from the onset of the disease $(63.3 \%)(\mathrm{p}=0.001)$ and the time of the first examination also occurred within 24 hours $(85.9 \%)(p=0.001)$. Conclusion: systemic arterial hypertension, ischemic stroke, moderate functional dependence, hospital management, and arrival time suggest that stroke patients may not be within the requirements of the temporal window to establish appropriate therapy for the control and evolution of ictus. This may indicate the need for public policies that contribute to the improvement of the SUS network, in its stroke care scope. Keywords: Stroke; Epidemiology; Public Health Surveillance; Health Education.
\end{abstract}




\section{INTRODUÇÃO}

O Acidente Vascular Cerebral (AVC) é causado pela interrupção do fornecimento de sangue ao cérebro, acarretando danos ao tecido cerebral, podendo levar à morte ${ }^{1}$. A carga global do AVC é de $59,2 \%$, sendo responsável por $5,7 \%$ dos anos vividos com deficiência em pessoas com 70 anos ou mais ${ }^{2}$. O AVC não é apenas um dos agravos mais comuns da atualidade, mas um problema de saúde pública global em termos de mortalidade, deficiência e demanda de custos médicos e sociais $^{3,4}$

Considerando-se os fatores de risco relacionados com o AVC, um estudo de rastreamento identificou dez fatores como significativos para o desenvolvimento da doença, tais como: tabagismo, uso regular de álcool, dieta pobre e inatividade; fatores individuais, tais como hipertensão arterial sistêmica (HAS), circunferência cintura-quadril elevada, diabetes; e fatores psicológicos, história de doença cardíaca e apolipoproteína elevada. Cerca de $54 \%$ dos casos de AVC no mundo são atribuíveis à $\mathrm{HAS}^{5}$. O Brasil tem comportamento similar à população mundial, com a HAS como fator de risco mais prevalente ${ }^{6}$.

Uma parcela significativa dos óbitos por AVC e das sequelas graves poderiam ser evitadas com medidas preventivas ou com prestação de socorro de forma correta e rápida. A identificação de alguns sinais e sintomas, como o deficit motor no hemicorpo, a paresia facial e a dificuldade para falar, aumenta muito as possibilidades do diagnóstico correto de $\mathrm{AVC}^{7}$. A tomografia computadorizada (TC) de crânio é indispensável na avaliação de emergência do paciente com AVC isquêmico agudo ${ }^{8}$.

O tratamento do paciente com AVC, além de depender diretamente do reconhecimento dos primeiros sinais e sintomas ${ }^{9}$, precisa da agilidade dos serviços de resgate, da estruturação dos serviços de emergência para rapidamente determinar o diagnóstico ${ }^{10}$ e da disponibilidade do tratamento trombolítico na rede pública ${ }^{11}$. No entanto, muitos pacientes e familiares não conhecem os sinais de alerta do AVC e, quando o fazem, não os caracterizam como uma emergência ${ }^{12}$, dessa forma, os pacientes podem não ser elegíveis para tratamento medicamentoso dentro de uma janela temporal de três horas após o início dos sintomas ${ }^{13}$.

O programa de aperfeiçoamento continuado no tratamento do AVC, Pacto AVC, no Brasil, tem a finalidade de capacitar profissionais envolvidos no atendimento e tratamento agudo do AVC, visando à organização e à eficácia da assistência emergencial ao paciente ${ }^{14}$. Destina-se a instituições de saúde em diferentes níveis de complexidade, sejam hospitais de nível secundário ou terciário ou na rede primária de atendimento à saúde, entretanto, é preciso avaliar se a implementação do programa tem sido eficiente, principalmente na região nordeste brasileira. Então, o objetivo do estudo foi avaliar dados epidemiológicos, clínicos e funcionais dos pacientes com AVC na chegada em um hospital público do nordeste brasileiro e verificar as condutas adotadas no primeiro atendimento dos pacientes.

\section{MÉTODO}

A pesquisa foi realizada por uma iniciativa do Programa de Diagnóstico e Intervenção no AVC (Prodiavc), desenvolvido pelo Departamento de Fisioterapia da Universidade Federal do Rio Grande do Norte (UFRN) e aprovada pelo Comitê de Ética em Pesquisa da UFRN sob o protocolo $n^{\circ}$ 193/06. 


\section{Amostra}

Foi realizado um estudo epidemiológico com uma amostra composta por 433 pacientes $(229$ mulheres e 204 homens), selecionados de acordo com os seguintes critérios de inclusão: AVC do tipo unilateral, de ambos os sexos, a partir de 18 anos de idade, analfabetos e escolarizados, que tinham condições neurológicas de responder aos questionários e com até três dias de lesão cerebral.

\section{Procedimentos}

A pesquisa foi realizada em um hospital público de Natal/RN, por meio do preenchimento do Step 1, que é um instrumento de base epidemiológica direcionado ao AVC, elaborado pela Organização Mundial da Saúde (OMS) e aplicado em ambiente hospitalar ${ }^{15}$. Por intermédio do Step 1, foram colhidas informações sobre fatores sociodemográficos (nome, sexo e escolaridade) e aspectos clínicos (fatores de risco e tipo do AVC). Outros dados registrados foram: tempo entre o AVC e a admissão no hospital, forma de condução ao hospital, local de atendimento imediato, local de internação, tempo de realização do primeiro exame e tipo de exame realizado.

Esse levantamento transversal se dava mediante vistoria nos prontuários dos indivíduos que ocupavam os leitos no local e que estavam até o seu terceiro dia pós-AVC. Apenas os indivíduos com quadro confirmado de AVC eram elegíveis para participação na pesquisa. Antes do início do experimento, todos os participantes, pacientes e/ou cuidadores foram devidamente esclarecidos acerca dos procedimentos da pesquisa e orientados a assinar um Termo de Consentimento Livre e Esclarecido. O questionário foi aplicado em um único momento com o paciente, tendo como período total de coleta de dados de janeiro a dezembro de 2009.
Os pacientes também foram avaliados pela Escala de Rankin Modificada obedecendo a seguinte classificação: $0=$ Sem nenhum sintoma; $1=$ Sem incapacidade significativa apesar dos sintomas: pode fazer todas as atividades habituais; $2=$ Pequena incapacidade: incapaz de fazer todas as atividades habituais prévias, mas capaz de se cuidar sem ajuda; 3= Moderada incapacidade: necessita de alguma ajuda, mas anda sozinho; 4= Moderada incapacidade: incapaz de andar sozinho e de realizar higiene corporal sem ajuda; 5= Grave incapacidade: acamado, incontinente e necessita de constantes cuidados da enfermagem.

\section{Análise estatística}

Os dados foram analisados por meio do programa SPSS 22.0 (Statistical Package for the Social Science), com nível de significância de 5\%. Foi utilizado o teste Qui-quadrado para verificar diferenças nas frequências absoluta e percentual das variáveis estudadas.

\section{RESULTADOS}

Pela análise dos fatores sociodemográficos, não foi encontrada diferença significativa na frequência de indivíduos do sexo feminino e masculino $(\mathrm{p}=0,23)$, entretanto, foram observadas diferenças significativas nas demais variáveis avaliadas: faixa etária e escolaridade. A maior frequência de AVC foi encontrada na faixa etária de 70 anos a 89 anos $(\mathrm{p}=0,001)$, e a maioria dos pacientes não tinha estudo formal $(p=0,001)$ (Tabela 1$)$. 


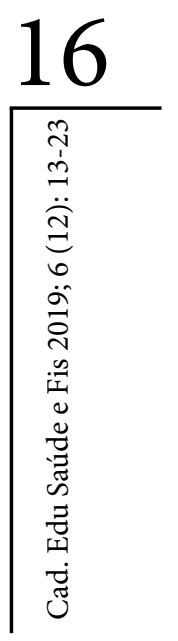

Tabela 1. Variáveis sociodemográficas dos pacientes com AVC.

\begin{tabular}{lll}
\hline Variáveis & N & \% \\
\hline Sexo & & \\
\hline Masculino & 204 & 47,1 \\
Feminino & 229 & 52,9 \\
\hline Idade & & \\
\hline$\geq 29$ anos & 6 & 1,4 \\
30 e 49 anos & 64 & 14,8 \\
50 e 69 anos & 166 & 38,3 \\
70 e 89 anos & 177 & 40,9 \\
$\leq 90$ anos & 20 & 4,6 \\
\hline Escolaridade & & \\
\hline Sem estudo formal & 178 & 41,2 \\
Fundamental incompleto & 126 & 29,2 \\
Fundamental completo & 71 & 16,4 \\
Médio incompleto & 24 & 5,5 \\
Médio completo & 25 & 5,7 \\
Superior completo & 7 & 1,6 \\
Pós-graduado & 2 & 0,4 \\
\hline
\end{tabular}

Os pacientes avaliados apresentaram diversos fatores de risco, entretanto, a HAS foi o mais frequente $(\mathrm{p}=0,001)$. $\mathrm{O}$ AVC isquêmico foi o de maior frequência na amostra estudada $(\mathrm{p}=0,001)$; e o perfil de dependência funcional, de acordo com a Escala de Rankin Modificada, mostrou maior frequência de pacientes com incapacidade moderada (incapaz de andar sozinho e de realizar higiene corporal sem ajuda) $(\mathrm{p}=0,001)$ (Tabela 2$)$. 
Tabela 2. Variáveis clínicas dos pacientes com AVC

\begin{tabular}{lll}
\hline Variáveis & N & \% \\
\hline Fatores de risco & & \\
\hline Hipertensão arterial & 370 & 85,4 \\
Diabetes & 149 & 34,4 \\
Cardiopatia & 100 & 23,1 \\
Dependência de nicotina & 219 & 50,5 \\
Consumo de álcool & 170 & 39,2 \\
Aids & 1 & 0,2 \\
Tuberculose & 9 & 2,1 \\
Câncer & 20 & 4,6 \\
Outro & 228 & 52,6 \\
\hline Tipo de AVC & & \\
\hline AVC Isquêmico & 297 & 68,5 \\
Hemorragia intraparenquimatosa & 47 & 10,8 \\
Hemorragia subaracnóidea & 36 & 8,3 \\
Outro & 49 & 11,3 \\
Desconhecido & 5 & 1,1 \\
\hline Rankin & & \\
\hline Sem incapacidade significativa & 27 & 6,2 \\
Pequena incapacidade & 45 & 10,4 \\
Moderada incapacidade: anda sozinho & 28 & 6,4 \\
Moderada incapacidade: incapaz de andar sozinho & 211 & 48,8 \\
e de realizar higiene corporal sem ajuda. & \multicolumn{2}{|c}{} \\
Grave incapacidade & 122 & 28,2 \\
\hline
\end{tabular}

Ao avaliar o tempo entre o aparecimento dos primeiros sinais e sintomas do AVC e a admissão no hospital, verificou-se maior frequência de pacientes que chegaram com menos de 24 horas $(\mathrm{p}=0,001)$, sendo trazidos mais frequentemente de outra unidade hospitalar do estado $(\mathrm{p}=0,001)$. O local de atendimento imediato mais registrado foi a emergência de outro hospital $(p=0,001)$, coerente com a forma de condução (transferência de outro hospital), sendo todos internados na Enfermaria de Neurologia. O tempo de realização do primeiro exame se deu mais nas primeiras 24 horas $(\mathrm{p}=0,001)$, tendo todos os pacientes se submetido à $\mathrm{TC}$ ( Tabela 3). 


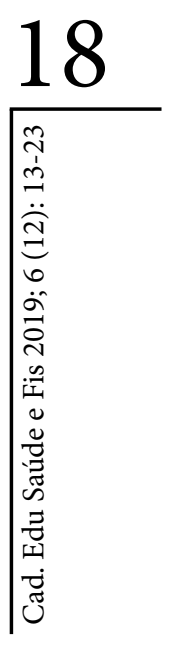

Tabela 3. Características dos pacientes com AVC na chegada ao hospital e primeiro atendimento

Variáveis
Tempo entre o AVC e a admissão no hospital
Menos de 24 horas

Menos de 24 horas

$274 \quad 63,3$

Mais de 24 horas

$159 \quad 36,7$

Forma de condução ao hospital

Resgate de emergência

$117 \quad 27,0$

Trazido por outrem

$99 \quad 22,9$

Já estava no HU por outro motivo

20,5

Transferido de outro hospital

$215 \quad 49,6$

\section{Local de atendimento imediato}

Pela Emergência do HU

Por equipe interna do $\mathrm{HU}$

$7 \quad 1,6$

Pela Emergência de outro hospital

$8 \quad 1,8$

Por equipe interna de outro hospital

$215 \quad 49,7$

Por equipe interna de posto de saúde

$29 \quad 6,7$

\section{Tempo de realização do $1^{\circ}$ exame}

Primeiras 24 horas

Entre 24 horas e 7 dias

Entre 8 dias e 14 dias

$3 \quad 0,6$

Desconhecido

10,2

\section{Tipo de exame realizado}

Tomografia Computadorizada

$433 \quad 100$

Ressonância Magnética

$5 \quad 1,1$

Angiografia

$11 \quad 2,5$

Outro

$300 \quad 69,2$

HU - Hospital Universitário 
Os pacientes que deram entrada no hospital vieram todos da mesma mesorregião do $\mathrm{RN}$, a mesorregião do Leste Potiguar, que tem 25 municípios (1.532.817 hab.), entre eles, a capital do estado, Natal, com 803.739 habitantes. $\mathrm{O}$ maior número de casos foi registrado advindo do município de Parnamirim (202.456 hab.), que faz parte da Grande Natal e possui um hospital estadual de emergência (Figura 1). Natal conta com 28 estabelecimentos de saúde com atendimento de emergência, porém o Hospital Monsenhor Walfredo Gurgel, local deste estudo, é tido como referência.

\section{DISCUSSÃO}

De acordo com a análise realizada, foi verificada maior predominância de AVC na faixa etária de 70 anos a 89 anos, o que corresponde aos dados encontrados na literatura ${ }^{17}$. Devido à proporção da população mundial com idade acima de 65 anos, é esperado um aumento de casos de AVC em todas as regiões do mundo, e isso é especialmente verdadeiro nos países em fase de desenvolvimento econômico. Dados da OMS estimam que o número projetado de mortes por doenças cerebrovasculares no mundo passará para 7,8 milhões em $2030^{18}$.

No presente estudo, verificou-se uma frequência de $41,2 \%$ de indivíduos sem estudo formal. A literatura mostra que o baixo nível de escolaridade, que pode servir como marcador de status socioeconômico, é um fator de risco importante para dependência funcional ${ }^{19} \mathrm{e}$ está ligado a um risco aumentado de morte após o $\mathrm{AVC}^{20}$.

No que se refere aos aspectos clínicos, foi encontrada maior frequência de AVC isquêmico, confirmando os achados da literatura, inclusive em estudo anterior no $\mathrm{RN}^{21}$. O AVC isquêmico também é mais frequente em indivíduos mais velhos, muitas vezes levando à hospitalização prolongada, à invalidez ou à morte ${ }^{21,22}$. Entre os fatores de risco modificáveis, a
HAS foi a mais evidenciada quando comparada aos demais fatores de risco, assim como encontrado na literatura ${ }^{15}$.

O quadro clínico apresentado mostra que $48,8 \%$ tinham incapacidade funcional moderada, com incapacidade de marcha e dependência para atividade de vida diária "higiene pessoal", sendo esta uma forte indicação para a necessidade do processo de reabilitação. Um estudo evidenciou que pacientes admitidos na reabilitação, dentro dos primeiros 30 dias do evento, apresentaram maiores ganhos funcionais e duração mais curta de permanência no hospital do que os que não realizaram nesse mesmo tempo ${ }^{23}$. Pessoas com AVC e limitações funcionais apresentaram menor satisfação com a vida do que as pessoas com AVC sem essas limitações, e isso se deve aos deficit motores e limitações nas suas atividades de vida diária (AVD) mostrando que a retenção de atividades e reintegração na comunidade são fatores essenciais para o sucesso da participação social ${ }^{24}$. Dessa forma, deve haver um esforço dos gestores de saúde para que a reabilitação seja iniciada o mais precocemente possível.

A Sociedade Brasileira de Doenças Cerebrovasculares ${ }^{25}$ recomenda diversos passos sequenciais a serem seguidos para um atendimento rápido e sistematizado, obedecendo a uma janela terapêutica para o tratamento do AVC. Estes são: reconhecimento dos sinais e sintomas, acesso ao cuidado, transporte ao hospital com TC, diagnóstico rápido no departamento de emergência, interpretação da TC, critérios de inclusão e tratamento. $\mathrm{O}$ presente estudo procurou basear-se nesses itens a serem avaliados, os quais são considerados no instrumento Step 1 da OMS.

$\mathrm{Na}$ amostra estudada, constatouse que o tempo decorrido entre o AVC e a admissão hospitalar foi inferior a 24 horas em $63,3 \%$ dos pacientes. É possível que alguns desses pacientes não tenham chegado ao hospital em um intervalo de três horas, o que certamente inviabilizaria a indicação do tratamento trombolítico para os pacientes com AVC isquêmico, 
caso o hospital o tivesse disponível. Os pacientes que chegam precocemente ao hospital tendem a ser aqueles que reconhecem os sinais e sintomas, dirigindo-se rapidamente a uma unidade de saúde ${ }^{12}$. Nesse sentido, os programas educacionais combinados envolvendo os serviços públicos, profissionais da saúde e a população devem ser considerados como prioridades.

O RN possui 167 municípios distribuídos em 4 mesorregiões. Os pacientes que chegaram ao hospital público durante a pesquisa vieram na grande maioria do Leste Potiguar, onde se localiza a cidade de Natal (capital do estado). Entretanto, o número de encaminhamentos advindos de municípios próximos a capital (mesma mesorregião) sugere que esse resultado pode estar relacionado com as desvantagens socioeconômicas da população. Os serviços de saúde dos municípios não conseguem suprir as necessidades da população, aumentando a demanda de pacientes para a capital, por consequência, causando superlotação na unidade.

No registro dos Estabelecimentos de Saúde de Natal, segundo os dados do Instituto Brasileiro de Geografia e Estatística (IBGE) ${ }^{26}$, foram identificados 88 estabelecimentos públicos, sendo 7 federais, 12 estaduais e 69 municipais, o que é pouco quando comparado com 335 estabelecimentos privados. Ainda segundo o IBGE, a capital possui 7 estabelecimentos com emergências em Neurocirurgia, 14 serviços de saúde públicos com internação e 3 estabelecimentos públicos com apoio à diagnose e terapia.

Quanto aos exames de imagem disponíveis, o IBGE registrou 18 Tomógrafos e 5 equipamentos de Ressonância Magnética. Nesse sentido, o acesso aos sistemas de saúde pelos pacientes com baixa condição econômica é precário, considerando que a capital acumula demandas de todo o estado. Mesmo assim, em relação à realização de exames de imagem, no período da pesquisa, todos os pacientes admitidos no hospital da pesquisa realizaram $\mathrm{TC}$, sendo uma rotina do hospital e nas primeiras 24 horas. Esses dados são, então, indicadores importantes para os órgãos governamentais, a fim de que possam avaliar medidas que contribuam para a melhoria dos serviços de saúde disponíveis para a população, não só no hospital de referência como em toda sua rede de atenção à saúde.

Outro aspecto importante é o local de atendimento imediato dos pacientes. No estudo realizado, vimos que a maior frequência foi o atendimento pela equipe interna de outro hospital, e, em seguida, encaminhados para o hospital da pesquisa e internados na Enfermaria de Neurologia, ou seja, esses pacientes não foram atendidos em uma Unidade de AVC, que é especializada para esse tipo de condição, porque em Natal não existe esse serviço; eles receberam atendimento com outros pacientes de diferentes patologias e vítimas de traumas, sugerindo que pode haver um significativo atraso na determinação do diagnóstico deles. Mesmo nos municípios em que ainda não se conta com Unidades de AVC, deve haver uma capacitação ampla e irrestrita por parte dos profissionais de saúde das unidades de emergência para reconhecimento imediato de um possível quadro de AVC e condutas necessárias.

No Brasil, poucos hospitais públicos dispõem de um serviço especializado para manejar o AVC agudo. A proposta de implantação de Unidades Vasculares nos serviços de emergência, de forma bem estruturada com leitos equipados e equipe treinada, seria a condição adequada para os sistemas de saúde ${ }^{27}$. Outra solução para o atendimento do AVC é a utilização da telemedicina, um sistema de teleconferência que permite a avaliação do paciente pela internet, de forma a poder colher a sua história, ter acesso aos seus exames neurológicos e elaborar o seu diagnóstico clínico. A telemedicina tem o potencial para melhorar a qualidade do cuidado ${ }^{28}$.

O resultado encontrado no presente estudo, de que o primeiro exame foi realizado dentro das primeiras 24 horas, também não garante que os casos de AVC 
isquêmico tenham sido diagnosticados nas primeiras três horas após o início da doença, o que também poderia comprometer a realização de um diagnóstico diferencial e estabelecimento do tratamento dentro da janela temporal.

As limitações encontradas para o desenvolvimento da pesquisa foram: a impossibilidade da coleta de dados quando o paciente se encontrava na Unidade de Terapia Intensiva e a dificuldade de acesso aos exames de imagem. No entanto, apesar dessas limitações, os achados apontam de forma significativa o perfil epidemiológico, clínico e funcional dos pacientes com AVC, ao chegarem a um hospital público de emergência na cidade de Natal/ RN (Figura 1).

Figura 1. Modelo esquemático do perfil dos pacientes com AVC referente à chegada ao hospital, à conduta adotada e às condições clínicas apresentadas, no período de janeiro a dezembro de 2009, na cidade de Natal/RN

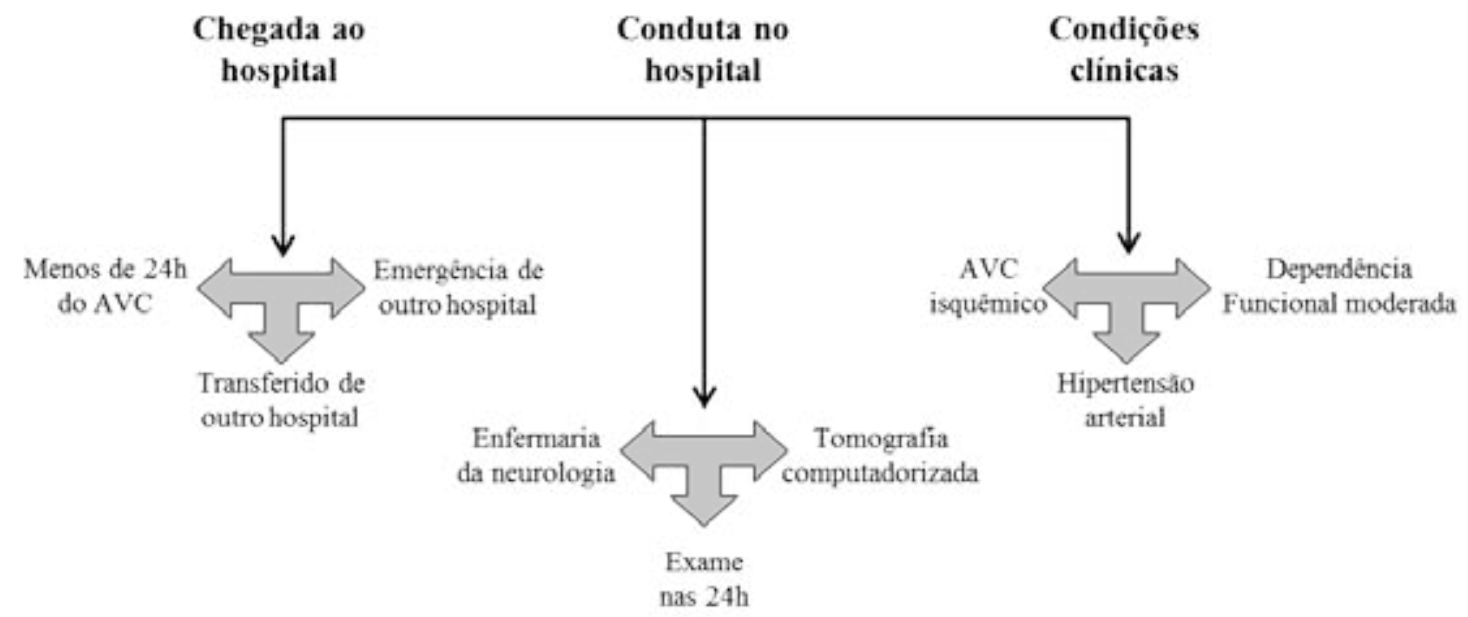

\section{CONCLUSÕES}

Conclui-se que a HAS, o AVC do tipo isquêmico, a dependência funcional moderada, a forma de condução ao hospital e o tempo de chegada sugerem que os pacientes com AVC podem não estar dentro dos requisitos da janela temporal para que se institua uma terapêutica adequada para o controle e a evolução do ictus, o que pode indicar a necessidade de políticas públicas que contribuam para o aperfeiçoamento da rede SUS, na sua linha de cuidado ao AVC. Em paralelo, a faixa de idade elevada e a baixa escolaridade refor- çam a dificuldade do atendimento inicial e da adesão ao tratamento necessário.

Nesse sentido, os programas educacionais combinados envolvendo os serviços públicos, os profissionais da saúde e a população podem abranger estratégias para reduzir o atraso e levar ao aumento da eficácia do tratamento do AVC. Assim, minimizar-se-ia a dificuldade no reconhecimento dos sinais e sintomas do AVC (educação em saúde), assim como a precariedade em atender à demanda em municípios vizinhos, com a implantação de Unidades de AVC no RN. 


\section{REFERÊNCIAS}

1. World Health Organization. Health topics - Stroke, Cerebrovascular accident. [Internet]. [Acessado 2016 set 27]. Disponível em: http://www.who.int/ topics/cerebrovascular_accident/en/.

2. GBD: Global Burden of Disease. IHME: Institute for Health Metrics and Evaluation. GBD compare data visualization [Internet]. Seattle: University of Washington, 2016 [cited 2017 Dec 7]. Available from: http://vizhub.healthdata. org/gbd-compare/

3. Byeon $\mathrm{H}$, Koh HW. The relationship between communication activities of daily living and quality of life among the elderly suffering from stroke. J Phys Ther Sci 2016; 28(5):1450-1453.

4. Abdul Aziz AF, Mohd Nordin NA, Ali MF, Abd Aziz NA, Sulong S, Aljunid SM. The integrated care pathway for post stroke patients (iCaPPS): a shared care approach between stakeholders in areas with limited access to specialist stroke care services. BMC Health Serv Res 2017; 17(1):35.

5. O’Donnell MJ, Xavier D, Liu L, Zhang H, Chin SL, Raomelacini P, et al. on behalf of the INTERSTROKE investigators. Risk factors for ischaemic and intracerebral haemorrhagic stroke in 22 countries (the INTERSTROKE study): a case-control study. Lancet 2010; 376(9735):112-123.

6. Avezum A, Costa-Filho FF, Pieri A, Martinsy SO, Marin-Neto JA. Stroke in Latin America: burden of disease and opportunities for prevention. Global Heart 2015; 10(4):323-331.

7. Rede Brasil AVC. [Internet]. [Acessado 2016 out 12]. Disponível em: http://www. redebrasilavc.org.br.
8. Pontes-Neto OM, Silva GS, Feitosa MR, Figueiredo NL, Fiorot JA Jr, Rocha TN, et al. Stroke Awareness in Brazil - Alarming Results in a Community-Based Study. Stroke 2008; 39:292-296.

9. Conforto $\mathrm{AB}$, Paulo RB, Patroclo $\mathrm{CB}$, Pereira SL, Miyahara HS, Fonseca CB, et al. Stroke management in a university hospital in the largest South American city. Arq Neuropsiquiatr 2008; 66(2):308311.

10. Mosley I, Nicol M, Donnan G, Patrick I, Dewey H. Stroke symptoms and the decision to call for an ambulance. Stroke 2007; 38:361-366.

11. Albers GW, Amarenco P, Easton JD, Sacco RL, Teal P, American College of Chest Physicians. Antithrombotic and thrombolytic therapy for ischemic stroke: American College of Chest Physicians Evidence-Based Clinical Practice Guidelines (8th edition). Chest 2008; 133(Supl.1):630-669.

12. Bouckaert M, Lemmens R, Thijs V. Reducing prehospital delay in acute stroke. Rev Neurol 2009; 5:477-483.

13. Hacke W, Kaste M, Bluhmki E, Brozman M, Dávalos A, Guidetti D, et al. Thrombolysis with alteplase 3 to $4.5 \mathrm{~h}$ after acute ischemic stroke. N Engl J Med 2008; 359:1317-1329.

14. Ministério da Saúde. Secretaria de Atenção à Saúde. Departamento de Atenção Básica. Pacto de Indicadores da Atenção Básica: instrumento de negociação qualificador do processo de gestão do SUS. Rev Bras Saúde Matern Infant 2003; 3(2):221-224.

15. Goulart AC, Bustos IR, Abe IM, Pereira AC, Fedeli LM, Benseñor IM, et al. A stepwise approach to stroke surveillance in Brazil: the EMMA (Estudo de Mortalidade e Morbidade do Acidente Vascular Cerebral) study. Int J Stroke 2010; 5(4):284-289. 
16. Wilson JTL, Harendran A, Grant M, Baird T, Schulz UGR, Muir KW, et al. Improving the assessment of outcomes in stroke: use off a structured interview to assign grades on the modified Rankin scale. Stroke 2002; 33:2243-2246.

17. Copstein L, Fernandes JG, Bastos GA. Prevalence and risk factors for stroke in a population of Southern Brazil. Arq Neuropsiquiatr 2013; 71(5):294-300.

18. Mathers CD, Loncar D. Projections of global mortality and burden of disease from 2002 to 2030. PLoS Med 2006; 3:e442.

19. Fernandes TG, Goulart AC, SantosJunior WR, Alencar AP, Benseñor IM, Lotufo PA. Educational levels and the functional dependence of ischemic stroke survivors. Cad. Saúde Pública 2012; 28(8):1581-1590.

20. Sposato LA, Ioli P, Povedano G, Esnaola y Rojas MM, Saposnik G, Argentinean Neurological Society and ReNACer Investigators. Unemployment: a social risk factor associated with early ischemic stroke mortality? Results from the Argentinean National Stroke Registry (ReNACer). J Stroke Cerebrovasc Dis 2012; 21(8):679-683.

21. Martins Jr AN, Figueiredo MM, Rocha OD, Fernandes MA, Jeronimo SM, Dourado Jr ME. Frequency of stroke types at an emergency hospital in Natal, Brazil. Arq Neuropsiquiatr 2007; 65:1139-1143.

22. Pinter MM, Brainin M. Rehabilitation after stroke in older people. Maturitas 2012; 71:104-108.
23. Brasil. Ministério da Saúde. Secretaria de Atenção à Saúde. Departamento de Ações Programáticas Estratégicas. Diretrizes de atenção à reabilitação da pessoa com acidente vascular cerebral / Ministério da Saúde, Secretaria de Atenção à Saúde, Departamento de Ações Programáticas Estratégicas. - Brasília: Ministério da Saúde, 2013.

24. Smith DL. Does type of disability and participation in rehabilitation affect satisfaction of stroke survivors? Results from the 2013 behavioral risk surveillance system (BRFSS). Disabil Health J 2015; 8(4):557-563.

25. Moro CHC, Fábio SRC, Longo AL, Massaro AR, Oliveira Filho J, Vedolin L, et al. Programa de aperfeiçoamento continuado no tratamento do acidente vascular cerebral - Pacto AVC. 2. ed. Sociedade Brasileira de Doenças Cerebrovasculares; 2009.

26. Instituto Brasileiro de Geografia e Estatística (IBGE). Assistência Médica Sanitária 2009. Rio de Janeiro: IBGE, 2010.

27. Candelise L, Gattinoni M, Bersano A, Micieli G, Sterzi R, Morabito A, et al. Stroke-unit care for acute stroke patients: an observational follow-up study. Lancet 2007; 369:299-305.

28. Steinman M, Morbeck RA, Pires PV, Abreu Filho CAC, Andrade AHV, Terra JCC, et al. Impacto da telemedicina na cultura hospitalar e suas consequências na qualidade e segurança do cuidado. Einstein. $2015 ; 13(4): 580-6$. 